\title{
Mathematical modeling of physico-chemical processes in the polymerization of multicore cable products
}

\author{
E.V. Ivanova, G.V. Kuznetsov, and P.A. Strizhak \\ Tomsk Polytechnic University, 634050 Tomsk, Russia
}

\begin{abstract}
In this article the question of simulation of no stationary process in the polymerization of multilayer cable product is considered. The model allows predicting time of full polymerization rubber shell.
\end{abstract}

\section{Introduction}

Processes of curing of cable products assume their transmission from specialized furnaces with a temperature of heating of $450 \div 550 \mathrm{~K}$. It is considered to be achievement on all thickness of an insulating layer of a cable of demanded [1] extents of polymerization $(\varphi \approx 0.99)$.

The purpose of this work is research of differences of integrated characteristics of curing of singlecore and multicore cable products.

\section{Problem statement}

Numerical modeling is executed for the typical system presented in Fig. 1. It was supposed that the cable contains some isolated conductors (copper conductor) and the general cover (rubber). Reference temperature of the product $\mathrm{T}_{0}$ was accepted lower than an air temperature in the heating camera $\mathrm{T}_{\mathrm{h}}$.

It was taken into account air gaps near entrance sites $\left(z=0, R_{5}<r<R_{6}\right)$ and output $\left(z=Z_{1}\right.$, $R_{5}<r<R_{6}$ ) cable product from vulcanizations furnace. There are considered that surrounding air flows into camera $\left(z=0, R_{5}<r<R_{6}\right)$ with temperature $T_{c}=T_{0}$ and speed $w_{c}$. Mix of cold and hot air follows from outlet $\left(z=Z_{1}, R_{2}<r<R_{3}\right.$ ) with speed $w_{c}$. There are axisymmetric systems (Fig. 1).

The following assumptions were accepted:

1. Contact between cores and rubber is ideal.

2. Cable has a correctly cylindrical form.

3. There are cables fragment with ideal insulated ends.

4. Thermophysical characteristics core, cover and air independent from temperature.

\section{Mathematical model}

The mathematical model of heat and mass transfer can be formulated as a typical time-dependent differential equations for system "hot air - multicore cable" in cylindrical coordinates [2,3]. 


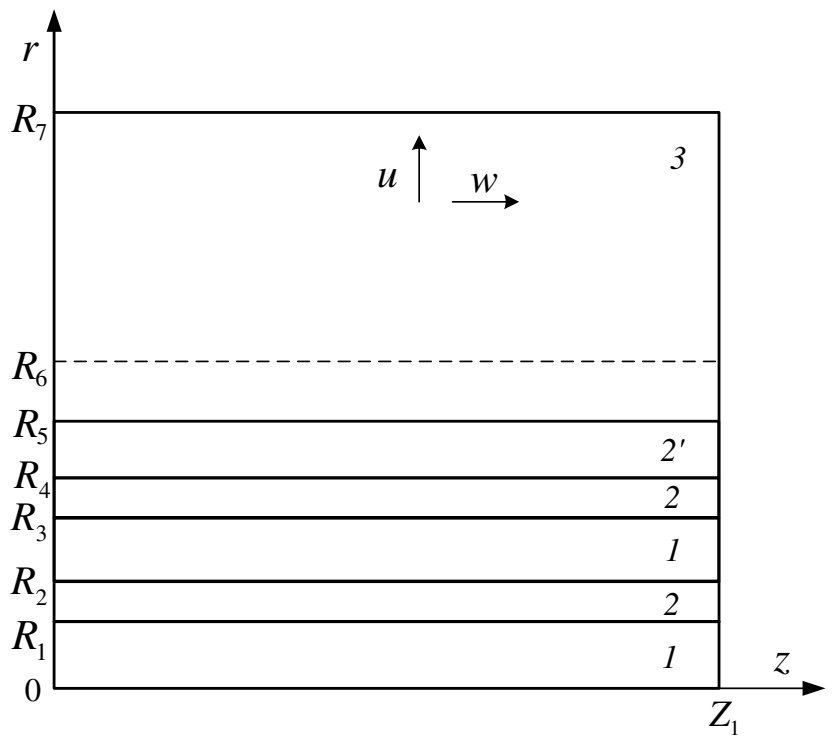

Figure 1. Scheme for solving the problem area: 1 - metal core, 2 - shell core, 2 ' - shell cable, 3 - hot air in furnace.

The heat equation for cores $\left(0<\mathrm{R}<\mathrm{Nr}_{1}, \mathrm{Nr}_{2}<\mathrm{R}<\mathrm{Nr}_{3}, 0<\mathrm{Z}<\mathrm{Nz}\right)$ :

$$
\frac{\partial T_{i}}{\partial t}=a_{i}\left[\frac{\partial^{2} T_{i}}{\partial R^{2}}+\frac{1}{R} \frac{\partial T_{i}}{\partial R}+\frac{\partial^{2} T_{i}}{\partial Z^{2}}\right], \quad \mathrm{i}=1,2
$$

The energy equation for core insulations $\left(\mathrm{Nr}_{1}<\mathrm{R}<\mathrm{Nr}_{2}, \mathrm{Nr}_{3}<\mathrm{R}<\mathrm{Nr}_{4}, 0<\mathrm{Z}<\mathrm{Nz}\right)$ :

$$
\frac{\partial T_{j}}{\partial t}=a_{j}\left[\frac{\partial^{2} T_{j}}{\partial R^{2}}+\frac{1}{R} \frac{\partial T_{j}}{\partial R}+\frac{\partial^{2} T_{j}}{\partial Z^{2}}\right], \quad j=1,2 .
$$

The energy equation for cable insulation shell $\left(\mathrm{Nr}_{4}<\mathrm{R}<\mathrm{Nr}_{5}, 0<\mathrm{Z}<\mathrm{Nz}\right)$ :

$$
\begin{aligned}
& \rho_{3} C_{3} \frac{\partial T_{3}}{\partial t}=\lambda_{3}\left[\frac{\partial^{2} T_{3}}{\partial R^{2}}+\frac{1}{R} \frac{\partial T_{3}}{\partial R}+\frac{\partial^{2} T_{3}}{\partial Z^{2}}\right]+q_{3} \rho_{3} \frac{d \varphi_{3}}{d t}, \\
& \frac{d \varphi_{3}}{d t}=\left(1-\varphi_{3}\right) k_{0} \exp \left[-\frac{E}{R_{t} T_{3}}\right] .
\end{aligned}
$$

The energy, motion and continuity equations for air in camera $\left(\mathrm{Nr}_{5}<\mathrm{R}<\mathrm{Nr}_{7}, 0<\mathrm{Z}<\mathrm{Nz}\right)$ :

$$
\begin{aligned}
& \frac{\partial T_{4}}{\partial t}+u \frac{\partial T_{4}}{\partial R}+w \frac{\partial T_{4}}{\partial Z}=a_{4}\left[\frac{\partial^{2} T_{4}}{\partial R^{2}}+\frac{1}{R} \frac{\partial T_{4}}{\partial R}+\frac{\partial^{2} T_{4}}{\partial Z^{2}}\right], \\
& \frac{\partial \omega}{\partial \tau}+u \frac{\partial \omega}{\partial R}+w \frac{\partial \omega}{\partial Z}=v_{4}\left[\frac{\partial^{2} \omega}{\partial R^{2}}+\frac{1}{R} \frac{\partial \omega}{\partial R}+\frac{\partial^{2} \omega}{\partial Z^{2}}\right]+\beta g \frac{\partial T_{4}}{\partial Z}, \\
& \frac{\partial^{2} \psi}{\partial Z^{2}}+\frac{1}{R} \frac{\partial \psi}{\partial R}+\frac{\partial^{2} \psi}{\partial R^{2}}=\omega .
\end{aligned}
$$


Initial $(\tau=0)$ conditions: $\mathrm{T}_{\mathrm{i}}=\mathrm{T}_{0}$ by $0<\mathrm{R}<\mathrm{Nr}_{1}, \mathrm{Nr}_{2}<\mathrm{R}<\mathrm{Nr}_{3}, 0<\mathrm{Z}<\mathrm{Nz} ; \mathrm{T}_{\mathrm{j}}=\mathrm{T}_{0}$ by $\mathrm{Nr}_{1}<\mathrm{R}<\mathrm{Nr}_{2}, \mathrm{Nr}_{3}<\mathrm{R}<\mathrm{Nr}_{4}, 0<\mathrm{Z}<\mathrm{Nz} ; \mathrm{T}_{3}=\mathrm{T}_{0}$ by $\mathrm{Nr}_{4}<\mathrm{R}<\mathrm{Nr}_{5}, 0<\mathrm{Z}<\mathrm{Nz} ; \mathrm{T}_{4}=\mathrm{T}_{v}$ by $\mathrm{Nr}_{5}<\mathrm{R}<\mathrm{Nr}_{7}, 0<\mathrm{Z}<\mathrm{Nz} ; \varphi=0,99$ by $\mathrm{Nr}_{1}<\mathrm{R}<\mathrm{Nr}_{2}, \mathrm{Nr}_{3}<\mathrm{R}<\mathrm{Nr}_{4}, 0<\mathrm{Z}<\mathrm{Nz} ; \varphi=\varphi_{0}$ by $\mathrm{Nr}_{4}<\mathrm{R}<\mathrm{Nr}_{5}, 0<\mathrm{Z}<\mathrm{Nz}$.

Boundary conditions $\left(0<\tau<\mathrm{t}_{p}\right)$ :

$$
\begin{aligned}
& \mathrm{Z}=0,0<\mathrm{R}<\mathrm{Nr}_{1}, \mathrm{Nr}_{2}<\mathrm{R}<\mathrm{Nr}_{3} \frac{\partial T_{i}}{\partial Z}=0 ; \\
& \mathrm{Z}=0, \mathrm{Nr}_{1}<\mathrm{R}<\mathrm{Nr}_{2}, \mathrm{Nr}_{3}<\mathrm{R}<\mathrm{Nr}_{4} \quad \frac{\partial T_{j}}{\partial Z}=0 ; \\
& \mathrm{Z}=0, \mathrm{Nr}_{4}<\mathrm{R}<\mathrm{Nr}_{5} \quad \frac{\partial T_{3}}{\partial Z}=0 ; \\
& \mathrm{Z}=0, \mathrm{Nr}_{5}<\mathrm{R}<\mathrm{Nr}_{6} T_{4}=T_{v}, \frac{\partial \psi}{\partial Z}=0, \frac{\partial \psi}{\partial R}=-w_{c} R ; \\
& \mathrm{Z}=0, \mathrm{Nr}_{6}<\mathrm{R}<\mathrm{Nr}_{7} \quad \frac{\partial T_{4}}{\partial Z}=0, \psi=0, \frac{\partial \psi}{\partial R}=0 ; \\
& \mathrm{Z}=\mathrm{Nz}, 0<\mathrm{R}<\mathrm{Nr}_{1}, \mathrm{Nr}_{2}<\mathrm{R}<\mathrm{Nr}_{3} \quad \frac{\partial T_{i}}{\partial Z}=0 ; \\
& \mathrm{Z}=\mathrm{NZ}, \mathrm{Nr}_{1}<\mathrm{R}<\mathrm{Nr}_{2}, \mathrm{Nr}_{3}<\mathrm{R}<\mathrm{Nr}_{4} \frac{\partial T_{j}}{\partial Z}=0 ; \\
& \mathrm{Z}=\mathrm{Nz}, \mathrm{Nr}_{4}<\mathrm{R}<\mathrm{Nr}_{5} \quad \frac{\partial T_{3}}{\partial Z}=0 \\
& \mathrm{Z}=\mathrm{Nz}, \mathrm{Nr}_{5}<\mathrm{R}<\mathrm{Nr}_{6} \quad T_{4}=T_{v}, \frac{\partial \psi}{\partial Z}=0, \frac{\partial \psi}{\partial R}=-w_{c} R \\
& \mathrm{Z}=\mathrm{Nz}, \mathrm{Nr}_{6}<\mathrm{R}<\mathrm{Nr}_{7} \frac{\partial T_{4}}{\partial Z}=0, \psi=0, \frac{\partial \psi}{\partial R}=0 ; \\
& \mathrm{R}=0,0<\mathrm{Z}<\mathrm{Nz} \frac{\partial T_{\mathrm{i}}}{\partial R}=0 \\
& \mathrm{R}=\mathrm{Nr}_{1}, 0<\mathrm{Z}<\mathrm{Nz}-\lambda_{i} \frac{\partial T_{\mathrm{i}}}{\partial R}=-\lambda_{j} \frac{\partial T_{j}}{\partial R}, T_{\mathrm{i}}=T_{\mathrm{j}} ; \\
& \mathrm{R}=\mathrm{Nr}_{2}, 0<\mathrm{Z}<\mathrm{Nz}-\lambda_{i} \frac{\partial T_{i}}{\partial R}=-\lambda_{j} \frac{\partial T_{j}}{\partial R}, T_{\mathrm{i}}=\mathrm{T}_{\mathrm{j}} ; \\
& \mathrm{R}=\mathrm{Nr}_{3}, 0<\mathrm{Z}<\mathrm{Nz}-\lambda_{i} \frac{\partial T_{i}}{\partial R}=-\lambda_{j} \frac{\partial T_{j}}{\partial R}, \mathrm{~T}_{\mathrm{i}}=\mathrm{T}_{\mathrm{j}} ; \\
& \mathrm{R}=\mathrm{Nr}_{4}, 0<\mathrm{Z}<\mathrm{Nz}-\lambda_{j} \frac{\partial T_{j}}{\partial R}=-\lambda_{3} \frac{\partial T_{3}}{\partial R}, \mathrm{~T}_{\mathrm{j}}=\mathrm{T}_{3} ; \\
& \mathrm{R}=\mathrm{Nr}_{5}, 0<\mathrm{Z}<\mathrm{Nz}-\lambda_{3} \frac{\partial T_{3}}{\partial R}=-\lambda_{4} \frac{\partial T_{4}}{\partial R}, \mathrm{~T}_{3}=\mathrm{T}_{4}, \psi=0, \frac{\partial \psi}{\partial Z}=w_{c} R ; \\
& \mathrm{R}=\mathrm{Nr}_{7}, 0<\mathrm{Z}<\mathrm{Nz} \mathrm{T}_{4}=T_{\mathrm{v}}, \psi=0, \frac{\partial \psi}{\partial Z}=0 .
\end{aligned}
$$




\section{EPJ Web of Conferences}

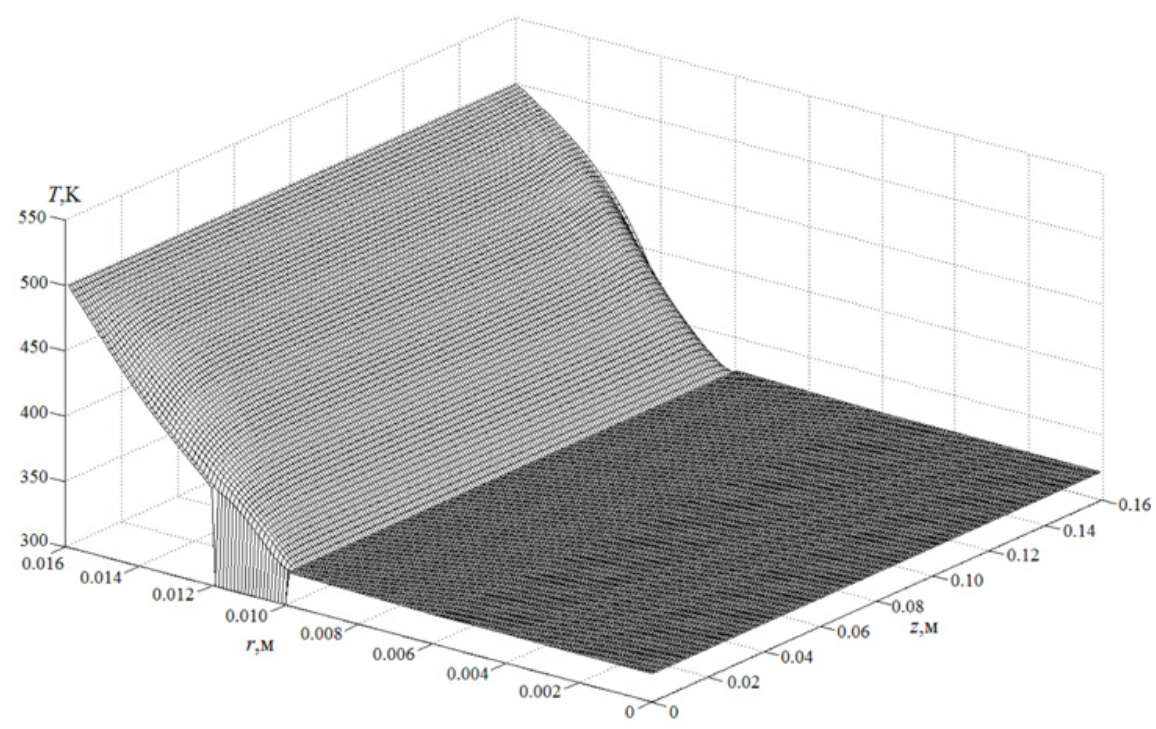

Figure 2. Temperatures field by full polymerization shell of multilayer cable $\left(\mathrm{t}_{\mathrm{p}}=774.6 \mathrm{~s}\right.$ and $\varphi \approx 0,99$ by $\mathrm{Nr}_{4}<\mathrm{R}<\mathrm{Nr}_{5}$ ).



Figure 3. Isotherms by full polymerization shell of multilayer cable $\left(t_{p}=774.6 \mathrm{~s}\right.$ and $\varphi \approx 0,99$ by $\mathrm{Nr}_{4}<\mathrm{R}<$ $\mathrm{Nr}_{5}$ ).

The system of time-dependent differential equations solved by finite difference method [4]. The difference analogues of differential equations solved by locally one-dimensional method and alternating direction method [4]. There are applied sweep method with using a four-point implicit scheme for solving dimensional difference equations [4]. The approximation of boundary conditions for Poisson equations and vortices equation are performed analogically [5]. 


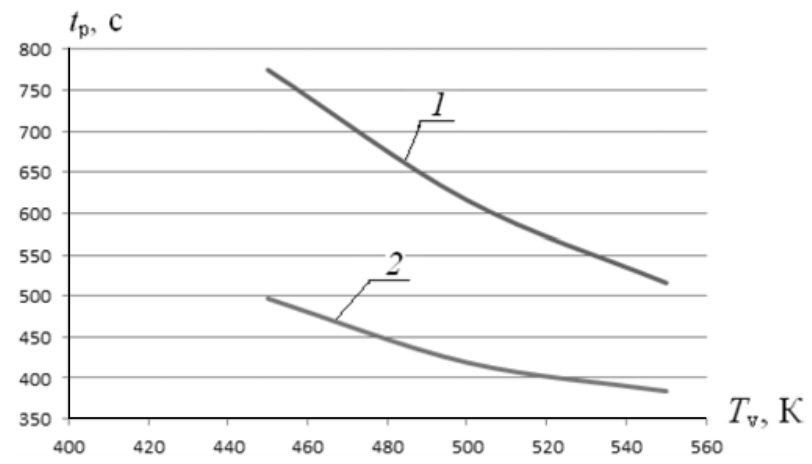

Figure 4. Dependence time of polymerization $t_{\mathrm{p}}$ from air temperature in camera $T_{v}: 1$ - multicore product, $2-$ single-core product.

\section{Results and discussion}

The results (Figs. 2-4) indicate about inexpedient of excerpts normative temperature for different structure of products. Correct choice of vulcanization mode $\left(T_{\mathrm{h}}, w_{\mathrm{c}}, t_{\mathrm{p}}\right)$ will minimize typical times of process and provide condition of uniform heating (without overheating, melting and surface defects).

There are found, that polymerization time of multicore cable's rubber cover are exceeds analogic parameters of single-core product. Our result indicates about accounting internal structure of cable product in selecting the vulcanization mode. There is need to support more times for multicore cable (accordingly, less speed of broach) as compared with single-core.

\section{Conclusion}

This model heat and mass transfer can use as prognostic to select of typical multicore cable's vulcanization mode and corresponding parameters (camera's temperature, allowable gap dimensions on in and out of camera, time of heating, speed of broaching).

This work supported by the Ministry of Education and Science Russian Federation (project 2.1321.2014).

\section{References}

[1] Lukomskaya A.I., Badenkov P.Ph., Kepersha L.M. Thermal curing basis of rubber productвые M.: Chemistry. 1972. 359 p. (Russia)

[2] P.J. Roache. Computational Fluid Dynamics, Hermosa Publishers, Albuquerque, N. Mex., 1982. $616 \mathrm{p}$

[3] Y. Jaluria, Natural Convection: Heat and mass transfer, Pergamon Press, 1980. 399 p

[4] Alexander A. Samarskii. The Theory of Difference Schemes. USA, Marcel Dekker, Inc., 2001. $788 \mathrm{p}$

[5] Ivanova E.V., Kuznetsov G.V. Numerical modeling of temperature fields in cylindrical articles during vulcanization // Journal Of Chemical and Petroleum Engineering, Volume 47, Issue 7-8, November 2011, P. 447-450 